\title{
Purification and Further Characterization of Human Mononuclear Cell Histamine-releasing Factor
}

\author{
Maria L. Baeza, Sesha Reddigari, Mary Haak-Frendscho, and Allen P. Kaplan \\ Division of Allergy, Rheumatology, and Clinical Immunology, Department of Medicine, State University of New York-Stony Brook, \\ Health Sciences Center, Stony Brook, New York 11794
}

\begin{abstract}
We have purified and further characterized a histamine releasing factor (HRF) derived from human mononuclear cells using gel-filtration HPLC, reverse-phase HPLC, anion exchange chromatography, and elution from SDS gels after electrophoresis. Considerable heterogeneity is seen, far exceeding that published in prior reports. Gel filtration HPLC yielded a major peak at molecular weight 30,000 and minor peaks at 50,000 and 12,000. Reverse-phase HPLC gave one major fraction in the void volume and an eluted peak at $50-60 \%$ acetonitrile. Accell QMA anion exchange HPLC revealed three peaks of activity; one in the void volume similar to that published previously using QAE-Sephadex, and peaks that eluted at 0.5 and $0.8 \mathrm{M}$ ammonium acetate, respectively. Electroelution following SDS-PAGE yielded peaks at MW 12,000 and 15-17,000 plus variable peaks at 25-27,000, 31-34,000, and 80-90,000 D. Using a combination of the aforementioned procedures, we have purified molecular species of HRF at 41,000 and 17,000 $\mathrm{D}$ to apparent homogeneity, as judged by SDS PAGE and autoradiography. Since human interleukin 3 and granulocyte-macrophage colony-stimulating factor possess histamine-releasing capability, it is clear that multiple cytokines can share this activity. However, the major HRF we isolate from human mononuclear cells appears, thus far, to be unique.
\end{abstract}

\section{Introduction}

Human basophils and mast cells can be stimulated to secrete histamine and other vasoactive substances upon interaction of antigen with surface bound IgE antibody (1) and upon stimulation with anaphylatoxins $\mathrm{C} 3 \mathrm{a}, \mathrm{C} 4 \mathrm{a}$, or C5a (2). However, in recent years it has become evident that basophils and mast cells can also be stimulated to release histamine by one or more factors derived from human mononuclear cells (3-5), platelets (6) neutrophils (7), lung macrophages $(8,9)$, and nasal washings (10). Basophils and mast cells may thereby participate in inflammatory reactions that have been initiated by other mechanisms.

Address reprint requests to Dr. A. Kaplan, Department of Medicine, Division of Allergy, Rheumatology and Clinical Immunology, SUNY-Stony Brook Health Sciences Center, Stony Brook, NY 11794-8160.

Received for publication 22 February 1988 and in revised form 21 November 1988.

J. Clin. Invest.

(c) The American Society for Clinical Investigation, Inc. 0021-9738/89/04/1204/07 \$2.00

Volume 83, April 1989, 1204-1210
We have previously reported the existence of one such histamine-releasing factor (HRF) ${ }^{1}$ with a major form at 30,000 mol wt and two charged species isoelectric at pI 6.9 and 7.3 (3). It is distinct from interleukin 1 , interleukin 2 , and $\gamma$ interferon as well as interleukins 4 and 5 (11), whereas human recombinant interleukin 3 (hr-IL3) and granulocyte-macrophage colony-stimulating factor (GM-CSF) were shown to possess histamine-releasing capability (11). Nevertheless, the major mononuclear cell-derived factor (MNC-HRF), as purified from cell supernatants, does not appear to correspond to these cytokines.

In this manuscript we demonstrate that human HRF is more heterogeneous than previously appreciated; multiple molecular forms were evident by gel filtration, ion exchange chromatography (on HPLC), and electroelution from SDS gels with molecular weights that range from 12,000 to 90,000 . By combining these methods we report the first procedure for the isolation and characterization of two of the major forms of human MNC-HRF of 41,000 and 17,000 mol wt, respectively.

\section{Methods}

Chloroform, 50-ml polypropylene tubes, ammonium acetate (HPLC grade), ammonium hydroxide, sodium hydroxide, glycerol, dialysis tubing, ethanol, cyanogen bromide and propanol (Fisher Scientific, Springfield, NJ); Ficoll-Hypaque, Sephadex G-25 (Pharmacia, Uppsala, Sweden); streptokinase/streptodornase (SK/SD; Lederle Laboratories, Pearl River, NY); RPMI 1640 with L-glutamine and Dulbecco's phosphate-buffered saline (Gibco Laboratories, Grand Island, NY); polyethylene-glycol, EDTA, sodium phosphate monobasic, sodium chloride, human IgG, potassium thiocyanate, piperazine- $N, N^{\prime}$-bis(2ethane sulfonic acid) (PIPES), deuterium oxide, human serum albumin, histamine hydrochloride, Tris-base, Hepes (Sigma Chemical Co., St. Louis, MO); YM100 and YM5 membranes (Amicon Corp., Danvers, MA); goat anti-human IgE (Kirkegaard \& Perry Lab, Inc., Gaithersburg, MD); $\left[{ }^{3} \mathrm{H}\right]$ histamine, $\left[{ }^{14} \mathrm{C}\right] S$-adenosyl methionine, $\left.{ }^{125}\right]$ (New England Nuclear Corp., Boston, MA); scintillation fluid, acrylamide and bisacrylamide (ICN, Radiochemicals, Irvine, CA); $60 \mathrm{~cm}$ $\times 7.5 \mathrm{~mm}$ TSK G3000 SW gel filtration HPLC column (Variant Corp., Sugar Land, TX), Accell quarternary methylamine (QMA) media, $\mu$ Bondapac reverse-phase C-18 column, trifluoroacetic acid, acetonitrile (Waters Associates, Milford, MA); iodo-beads, phenylisothio cyanate (PITC) reagent (Pierce Chemical Co., Rockford, IL); and triethylamine (Aldrich Chemical Co., Milwaukee, WI); were purchased as indicated. All reagents were of greater than $99 \%$ purity.

Cell source. Donors of leukopheresis packs were obtained from two sources: either normal volunteers, after informed consent, or patients undergoing cytopheresis according to an experimental protocol for the treatment of multiple sclerosis. The molecular characterization of the

1. Abbreviations used in this paper: HBS-HSA, Hepes-buffered salinehuman serum albumin; MNC-HRF, mononuclear cell-derived histamine-releasing factor; PITC, phenylisothiocyanate; PTH, phenylthiohydantoin; QMA, quarternary methylamine; SK/SD, streptokinase/ streptodornase. 
HRF described herein did not differ according to source, and the yields of activity appeared comparable.

Preparations of mononuclear cell supernatants. Leukopheresis packs of $\sim 200 \mathrm{ml}$ were diluted to $360 \mathrm{ml}$ in Dulbecco's PBS. $30 \mathrm{ml}$ of cellular suspension were layered over $15 \mathrm{ml}$ of Ficoll-Hypaque in Falcon 50-ml polypropylene tubes. The suspension was centrifuged for 15 $\min$ at $1,000 \mathrm{~g}$. The interface was aspirated, pooled, washed with PBS, and centrifuged at $400 \mathrm{~g}$ for $12 \mathrm{~min}$. After a second wash, the mixture was centrifuged at $150 \mathrm{~g}$ for $7 \mathrm{~min}$ to deplete platelets. The remaining MNC pellet was resuspended at a concentration of $25-45 \times 10^{6}$ cells/ $\mathrm{ml}$ in RPMI 1640 tissue culture medium. Cell viability was $>98 \%$ by trypan blue exclusion. SK/SD was added to the cell cultures at 13 $\mathrm{U} / 10^{6}$ cells. The cells were then cultured for $16-19 \mathrm{~h}$ at $37^{\circ} \mathrm{C}$ with $5 \%$ $\mathrm{CO}_{2}$ in Falcon $50 \mathrm{ml}$ conical polypropylene tubes. At the end of the culture period the conditioned media were pooled, centrifuged at $400 \mathrm{~g}$ for $15 \mathrm{~min}$, and clarified at $38,000 \mathrm{~g}$ for $20 \mathrm{~min}$. The supernatant was filtered through Amicon YM100 membranes and concentrated 50fold using Amicon YM5 membranes. PMSF was added to a final concentration of $0.1 \mathrm{mM}$ before freezing and the material was stored at $-20^{\circ} \mathrm{C}$. In separate experiments we demonstrated that this concentration of PMSF had no effect upon the release of histamine by HRF.

Basophil histamine release. The procedures for basophil isolation and histamine release have been described in detail (3). Briefly, the cells were prepared by dextran sedimentation $(0.6 \%$ dextran, $0.6 \%$ glucose, $0.02 \%$ EDTA), the basophil containing layer washed twice with Hepes buffered saline-human serum albumin (HBS-HSA), and the cells resuspended in HBS-HSA containing $2 \mathrm{mM} \mathrm{CaCl}_{2}, 2 \mathrm{mM}$ $\mathrm{MgCl}_{2}$, and $50 \% \mathrm{D}_{2} \mathrm{O} .20-50 \mu \mathrm{l}$ of each sample or PBS control were incubated with $3 \times 10^{6}$ cells in a total volume of $300 \mu \mathrm{l}$ for $40 \mathrm{~min}$ at $37^{\circ} \mathrm{C}$ and centrifuged at $1,000 \mathrm{~g}$ for $5 \mathrm{~min}$. The total basophil histamine content was determined by boiling one of the aliquots. The presence of $\mathrm{D}_{2} \mathrm{O}$ had no effect on the baseline histamine release and augmented the histamine release $2-2.5$-fold. Therefore it was routinely used to facilitate the assay and conserve material.

Histamine assay. The radioenzyme assay for histamine was performed using a modification of the method of Beaven et al. (12) as described (3). Standard curves for each assay were constructed by linear regression using a computer program written by Dr. M. Silverberg (SUNY, Stony Brook, NY) using an Apple Ile computer.

${ }^{125}$ I Radiolabeling. $20 \mu \mathrm{l}$ of sample were radiolabeled with ${ }^{125}$ I using iodo-beads as described by Markwell (13). One iodo-bead (Pierce Chemical Co.) was placed into each reaction vial with $0.1 \mathrm{mCi}$ of ${ }^{125} \mathrm{I}$ and incubated for $5 \mathrm{~min}$ at room temperature. $20 \mu \mathrm{l}$ of the protein sample and $30 \mu$ l of PBS were added to the reaction vial and further incubated for $15 \mathrm{~min}$ at room temperature. The iodo-bead was removed and the samples were passed through a Sephadex G-25 minicolumn (see below) to separate the iodinated protein from unreacted iodine.

Autoradiography. Iodinated protein $\left(1.4 \times 10^{5} \mathrm{cpm}\right)$ was subjected to SDS-PAGE on $12 \%$ gels. The gel was fixed, dried, and developed by exposure to X-omat-AR/film for $7 \mathrm{~h}$.

Protein analysis. Protein was determined by the Bradford method (14). 5-30 $\mu \mathrm{l}$ of the sample protein was placed into microtiter plates and the volume adjusted to $30 \mu \mathrm{l}$ with PBS. $200 \mu \mathrm{l}$ of Bradford reagent was added and the absorbance at $570 \mathrm{~nm}$ was measured after $10 \mathrm{~min}$. $30 \mu 1$ of PBS or serial concentrations of human IgG were used for the reagent blank and standard curve, respectively. For calculation of protein recovery (Table I) from column chromatograms we sum the protein content of each tube and divide by the protein applied. For recovery from gels, we take an aliquot from each eluate (5 $\mu$ l of 200$)$, add them together to one tube, lyophilize, resuspend into a fixed volume of PBS, and determine protein content. Absolute protein $\times 40 /$ protein applied to the gel $=$ protein recovery.

Recovery of activity is calculated as follows: the activity of an aliquot is determined, and (as above) converted to activity/ $\mu$ l and multiplied by the volume $(\mu 1)$ of the peak minus the activities of peaks are summed and this is divided by the activity applied to obtain a percent.
Table I. Protein and Activity Recovery

from Purification Procedures

\begin{tabular}{lll}
\hline \multicolumn{1}{c}{ Procedure } & $\begin{array}{l}\text { \% Protein } \\
\text { recovery* }\end{array}$ & $\begin{array}{l}\% \text { Activity } \\
\text { recovery }\end{array}$ \\
\hline GF-HPLC & $90-100$ & $90-100$ \\
RF-HPLC & $80-90$ & 40 \\
Accell-QMA Anion exchange HPLC & $90-100$ & $90-100$ \\
SDS-PAGE electroelution & $40-50$ & 40 \\
Sephadex G25 spun column & $90-100$ & $90-100$ \\
& & \\
\hline
\end{tabular}

* See Methods for calculation of recoveries.

Gel filtration by HPLC. Concentrated crude supernatants of SK/SD-stimulated MNC were applied to a $0.75 \times 60 \mathrm{~cm}$ TSK G3000 SW gel filtration HPLC column equilibrated with PBS pH 7.4 containing $0.3 \%$ PEG 8000 . The column was run at $0.5 \mathrm{ml} / \mathrm{min}$ and $1 \mathrm{ml}$ fractions were collected; $50 \mu \mathrm{l}$ of each fraction was assayed for histamine release.

Accell-QMA anion exchange chromatography. A $3.9 \mathrm{~mm} \times 15 \mathrm{~cm}$ stainless steel Waters column was packed with $2 \mathrm{~g}$ of Accell-QMA media, which gave a bed volume of $1.8 \mathrm{ml}$. The column was equilibrated with $0.02 \mathrm{M}$ ammonium acetate $(\mathrm{pH} 8)$ at a flow rate of 0.5 $\mathrm{ml} / \mathrm{min} .1 .5 \mathrm{ml}$ of highly concentrated crude supernatant of SK/SD stimulated MNC or material obtained from gel filtration was dialyzed overnight against the starting buffer and applied to the column. After a 15-min wash, a gradient was applied from 0.02 to $0.5 \mathrm{M}$ ammonium acetate for $20 \mathrm{~min}$. The column was washed with the $0.5 \mathrm{M}$ salt buffer for $15 \mathrm{~min}$ and a second gradient was applied from 0.5 to $1.0 \mathrm{M}$ ammonium acetate for $15 \mathrm{~min}$. After washing with $1.0 \mathrm{M}$ buffer a gradient was again applied from 1.0 to $3.0 \mathrm{M}$ buffer to strip the column. $1.5 \mathrm{ml}$ fractions were collected, lyophilized and resuspended in $300 \mu \mathrm{l}$ of PBS. $50 \mu \mathrm{l}$ of each fraction were passed through a Sephadex G-25 minicolumn equilibrated with PBS to remove any trace of ammonium acetate.

Desalting G25 minicolumns. To avoid any low molecular weight contaminant that could interfere with the basophil release assay, all the samples were desalted on Sephadex G-25 minicolumns by a modification of the procedure of Maniatis et al. (15). A single perforation was made at the bottom of 500- $\mu \mathrm{l}$ microfuge tubes with a $20 \mathrm{G}$ hypodermic needle and the bottom plugged with a small amount of glass wool. The columns were packed with Sephadex G-25 $(500 \mu l)$ in PBS (pH 7.4) and equilibrated with $50 \mu \mathrm{l}$ of the same buffer by centrifugation three times at $1,500 \mathrm{~g}$ for $4 \mathrm{~min}$. The samples $(50 \mu \mathrm{l})$ were then applied and centrifuged at $1,500 \mathrm{~g}$ for $4 \mathrm{~min}$.

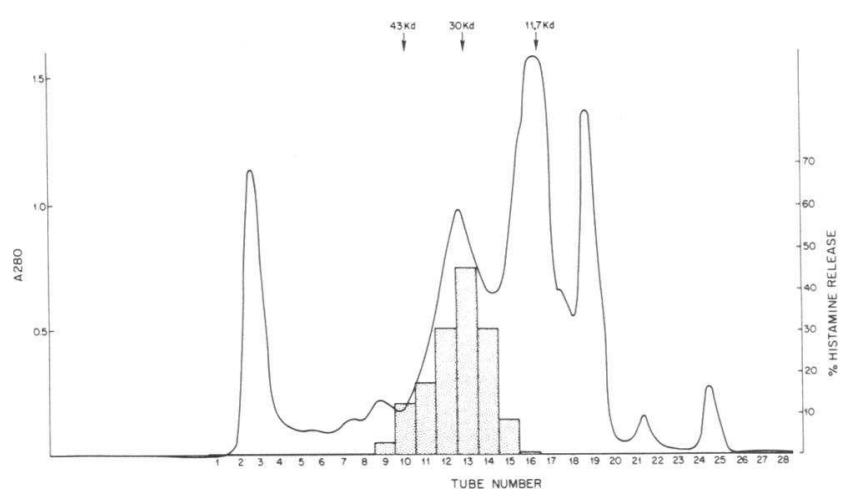

Figure 1. Gel filtration HPLC of human MNC-derived supernatant. The solid line is protein determined by optical density at $280 \mathrm{~nm}$. The percent histamine release is shown by the vertical bars; a peak at molecular weight 30,000 is seen. 


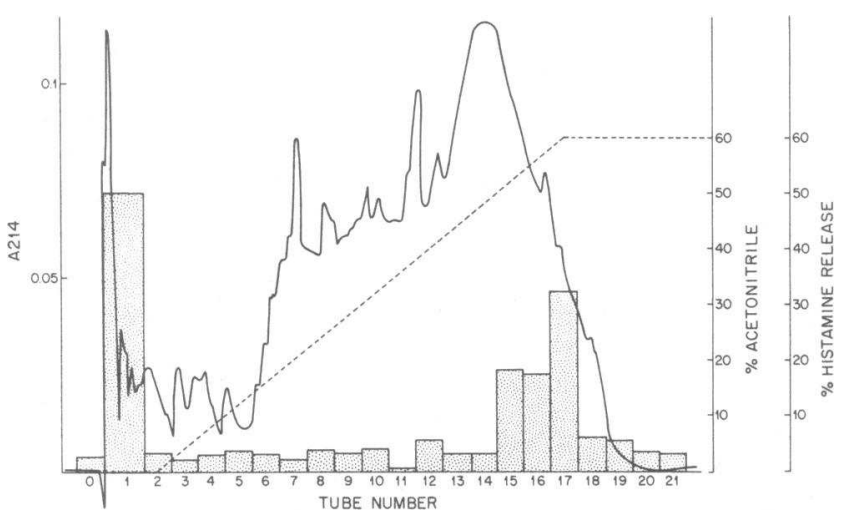

Figure 2. Reverse-phase HPLC of MNC-derived supernatant developed with an acetonitrile gradient. The optical density at $214 \mathrm{~nm}$ is given by the solid line and histamine release is shown by the vertical striped bars. Activity is seen in the void volume and at the end of the gradient.

Reverse-phase-HPLC. Reverse-phase HPLC was used to characterize and/or further purify HRF. The sample was clarified by centrifugation at $10,000 \mathrm{~g}$ and $200 \mu \mathrm{l}$ of crude concentrate were loaded onto a 3.9 $\times 30 \mathrm{~cm} \mu$ Bondapac $\mathrm{C}$ column. After a 5 -min wash with $0.1 \%$ trifluoroacetic acid in water, a gradient was applied with acetonitrile as limitbuffer for 30 min such that a concentration of $60 \%$ acetonitrile was reached. The column was then stripped with acetonitrile for 10 more $\mathrm{min}$. The flow rate was $1 \mathrm{ml} / \mathrm{min}$ and $2-\mathrm{ml}$ fractions were collected. The fractions were lyophilized, resuspended in $100 \mu \mathrm{l}$ of PBS, and $20 \mu \mathrm{l}$ of each fraction was assayed for basophil histamine release.

Electroelution after SDS-polyacrylamide gel electrophoresis. Crude supernatant or protein peaks obtained after anion exchange chromatography were analyzed and/or fractionated in 1.5- or 3-mm slab SDS-PAGE (12\% acrylamide) by the procedure of Laemmli (16). At the end of the run, the gel lanes containing the samples were sliced and each slice placed into a dialysis bag (3,500 mol wt cutoff) with $3 \mathrm{ml}$ of $25 \mathrm{mM}$ phosphate buffer, pH 9. Each dialysis bag (usually 33 ) was placed in an electroblotting tank (Bio-Rad Laboratories, Richmond, CA) containing the same buffer. 15-17 V were applied for $18 \mathrm{~h}$ at room temperature. The buffer was changed three times during the electroelution to remove all traces of SDS. Orientation of the bags was care- fully maintained. The samples were then dialyzed against 12 liters of $0.05 \mathrm{M}$ ammonium acetate $\mathrm{pH} 7.4$ for $4 \mathrm{~h}$ with one buffer change. The samples were filtered, lyophilized, resuspended in 200-250 $\mu$ l of PBS, and a portion passed over a Sephadex G-25 minicolumn before assay.

\section{Results}

To further characterize human MNC-HRF we first fractionated a pooled, concentrated SK/SD stimulated MNC supernatant by three different fractionation procedures; namely, gel filtration HPLC, reverse-phase HPLC, and Accell-QMA anion exchange chromatography on HPLC. When crude concentrated HRF was applied to gel filtration HPLC (Fig. 1), a single major peak of activity was found at an apparent molecular weight of 30,000 as had been reported previously (3). In some chromatograms, a small additional peak of activity was seen at either 12,000 or at $\sim 50,000 \mathrm{~mol}$ wt. These latter peaks are variable and only the 30,000 peak is consistently obtained. The recovery of both total protein as well as activity were between 90 and $100 \%$.

We then characterized the same crude material by reversephase HPLC using an increasing gradient of acetonitrile as described in Methods. The chromatogram obtained is shown in Fig. 2. About half the activity did not adhere to the column and was obtained in the effluent. A second peak of activity was eluted late in the gradient at a concentration of $50-60 \%$ acetonitrite. The protein recovery was $80 \%$ (Table I), however the activity recovered was considerably lower at $40 \%$. We therefore sought other methods that might separate the various forms of $\mathrm{HRF}$ with greater yield.

Fractionation on a Accell-QMA anion exchange HPLC column of crude supernatant was attempted next using a gradient of ammonium acetate interrupted with washes at 0.5 , 1.0 , and $3.0 \mathrm{M}$ concentration. Some HRF was routinely obtained in the effluent, a second peak of activity was obtained at $0.5 \mathrm{M} \mathrm{NaCl}$, and a third peak just preceding the 1.0 molar wash. The last two peaks were clearly separated by a trough and this was reproducible in eight successive columns that were run (Fig. 3). The recovery on Accell-QMA was 90-100\% for both protein and activity, and this was thought to be a

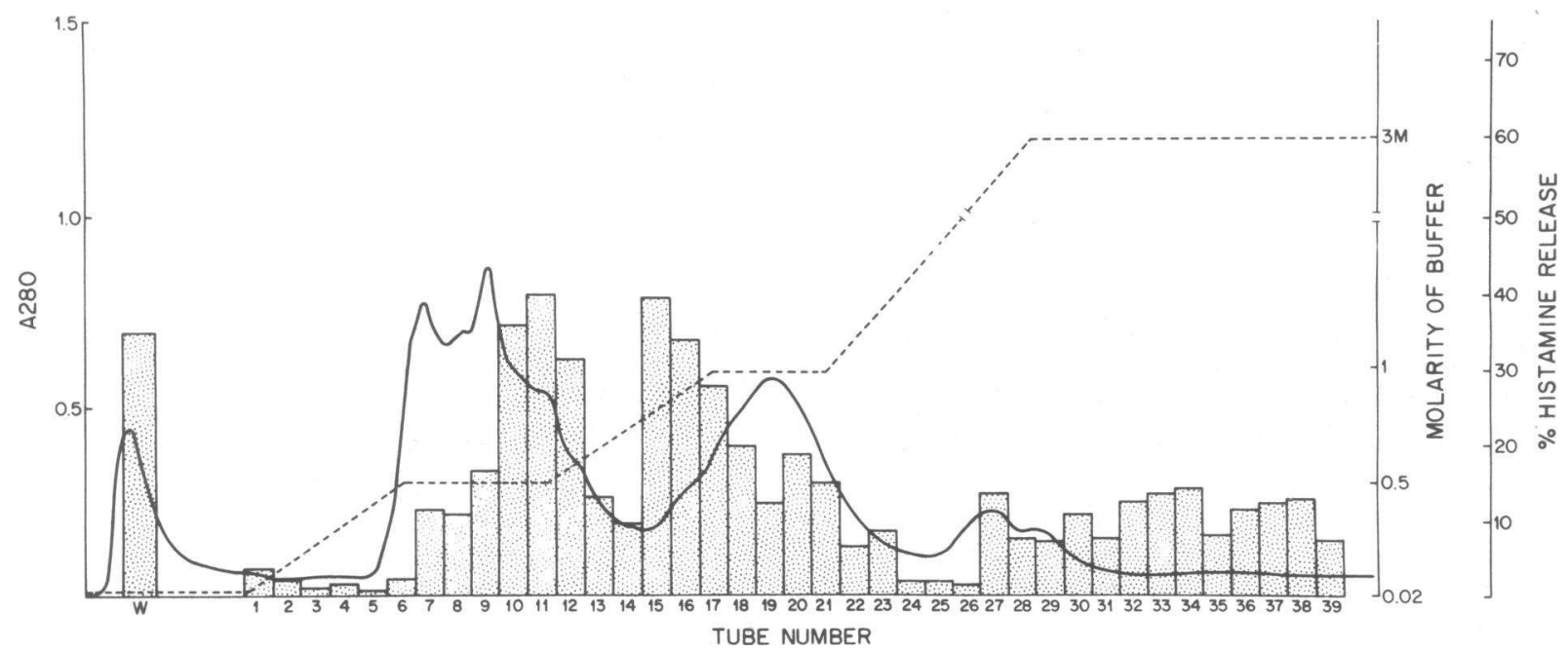

Figure 3. Accell-QMA anion exchange HPLC of MNC-derived supernatant containing HRF activity. Molarity of the buffer in gradients and wash phase is indicated by the dashed line. The optical density at $280 \mathrm{~nm}$ is shown by the solid line and percent histamine release by the vertical bars. Major areas of activity are in the void volume, during the $0.5-\mathrm{M}$ wash phase, and at the end of the $1.0-\mathrm{M}$ gradient. 

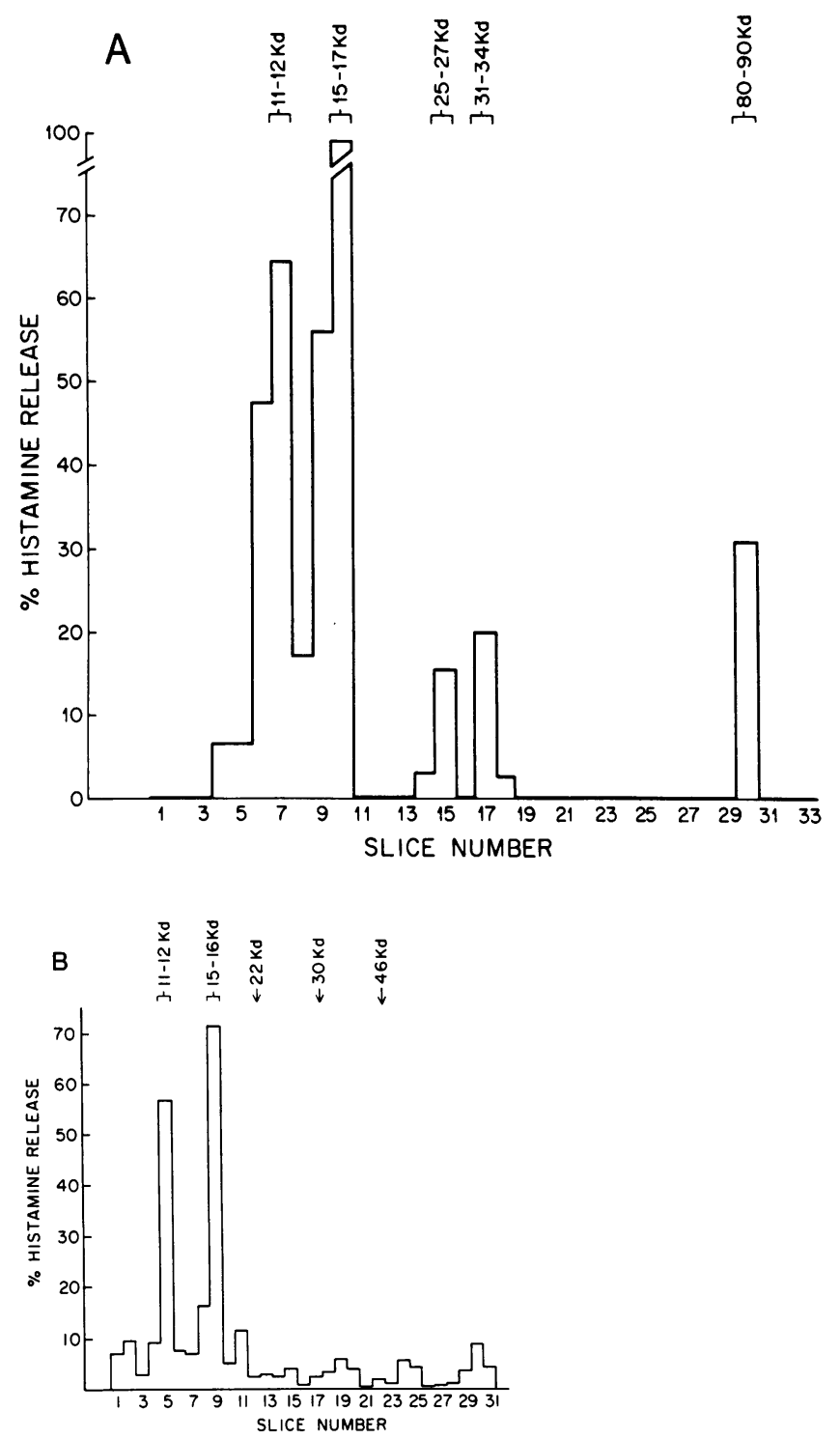

Figure 4. ( $A$ and $B$ ) SDS gel electrophoresis of two separate MNC supernatants applied directly to the gels. The gels were sliced, electroeluted, and fractions assayed for histamine release. The positive fractions are shown by the vertical bars and the corresponding molecular weights are given.

superior separation step when compared to reverse-phase HPLC. The Accell-QMA fractions were lyophilized, resuspended in PBS, and the aliquot to be assayed was passed over a Sephadex G-25 column to remove trace quantities of ammonium acetate. This step was critical since even small amounts of ammonium acetate cause nonspecific basophil histamine release. The recovery from such a column was $90-100 \%$ as long as the total protein applied was $>2 \mu \mathrm{g}$.

Before proceeding with successive chromatographic steps to obtain a purified product, we next wished to determine the mobility of crude HRF supernatant upon nondenaturing disk gels and SDS gels. After electrophoresis of $400 \mu \mathrm{l}$ of sample, the gels were cut into 4-mm slices and proteins were electroeluted. Each eluted fraction was dialyzed against ammonium acetate, lyophilized, resuspended in PBS, and an aliquot passed over a Sephadex G-25 column. The effluent was assayed for HRF. Generally, the activity recovered from SDS gels as well as nondenaturing gels was $40 \%$. The protein recovery was $40-50 \%$. Representative assays are shown in Fig. $4 \mathrm{~A}$ and $B$. Two major peaks of activity are shown at molecular weights of $11,000-12,000$ and $15,000-17,000$, as well as minor peaks at $25,000-27,000,31,000-34,000$, and 80,000 (Fig. $4 A$ ). Thus fractionation of the initial supernatant, which yields a major peak of 30,000 D upon gel filtration, is clearly a heterogeneous mixture that can be further resolved when assessed by SDS gel electrophoresis.

We then sequentially fractionated material in the following fashion. The peak obtained on gel filtration was fractionated by Accell-QMA anion exchange HPLC. Each of the three active peaks was then subjected to SDS gel electrophoresis and the eluates processed as described and assayed for HRF. As shown in Fig. 5, a single peak of HRF activity was obtained at a molecular weight of 15,000-17,000 when the second QMA fraction was assessed. This SDS eluate was then iodinated, resubjected to SDS gel electrophoresis, and developed by autoradiography (Fig. 6). A single band at $17,000 \mathrm{D}$ is seen. When the third peak from the Accell QMA anion exchange column was applied to SDS-gel electrophoresis (Fig. 7), a major peak was found at molecular weight 41,000 , and minor peaks at $7,000,11,000-12,000,14,000-17,000$ and $65,000-85,000$. This pattern was reproduced when three separate supernatants (three different donors) were subjected to sequential fractionation by gel filtration, Accell QMA anion chromatography, and the third peak subjected to SDS gel electrophoresis. This 41,000 moiety peak appears as a variable minor area of activity when crude supernatant is fractionated directly into SDS gels (Fig. 4), but its activity is prominent after the described purifcation procedure. The reason the apparent activity increases is not known. It is possible that one or more inhibitors are also present in the supernatant stage, which can act upon the factor itself or the responsiveness of basophils or that an activation step occurs in the course of purification. In Fig. 8 is shown a radioautogram of this iodinated peak and a single major band at 41,000 is detected.

A preliminary assessment of peak I reveals that it is far more heterogeneous than peak II or III; the predominant HRF is at $11,000-12,000 \mathrm{D}$, but we also find activity at 15,000 $18,000,40,000-45,000$, and $80,000-90,000$. The predominant 12,000 molecular weight factor was eluted from gels, and gave

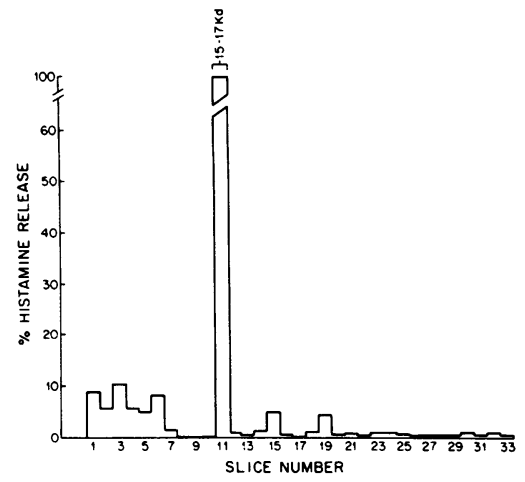

Figure 5. SDS gel electrophoresis of MNCHRF supernatant that was first subjected to gel filtration and AccellQMA anion exchange HPLC. The second peak from the QMA column was applied to the SDS-gel. The gel was sliced, electroeluted, and the fractions assayed for histamine release. The major activity was the 15,000 17,000 mol wt peak. 


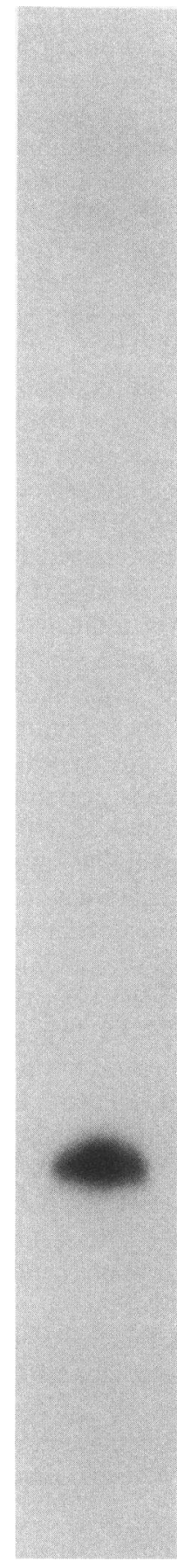

Figure 6. HRF obtained by electroelution after SDS gel electrophoresis of the Accell QMA peak II, was radiolabeled, subjected again to SDS gel electrophoresis and a autoradiogram obtained. A single band of purified material is seen, at 17,000 mol wt.

a single band upon silver stained SDS gels (not shown). It is clear that the aforementioned procedure can be used to purify each of the major forms of mononuclear cell-derived HRF. The recovery of protein and activity obtained with each purification step is summarized in Table I.

\section{Discussion}

Although HRF has been reported to be derived from a variety of cell sources, it has not been purified from any of them. In this manuscript we have developed methods to purify and characterize the material derived from human mononuclear

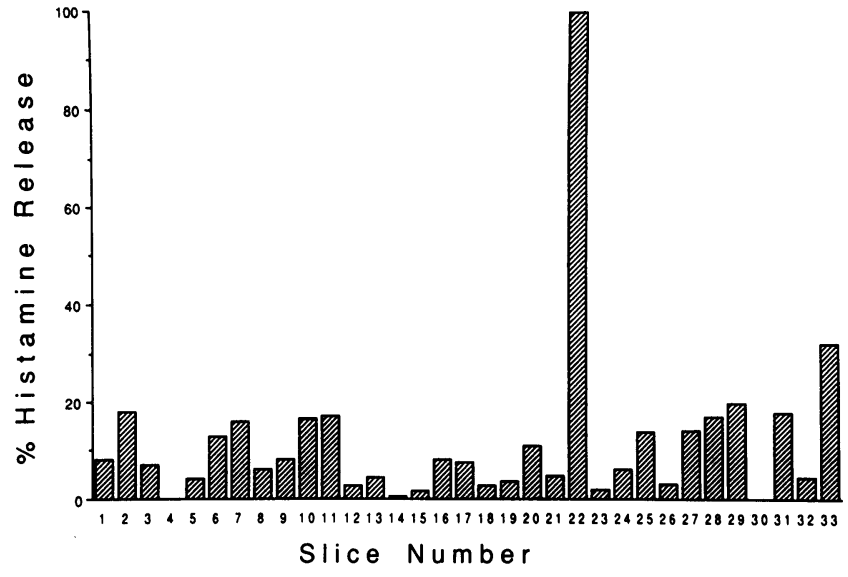

Figure 7. Protein electroelution of Accell QMA peak III from SDSPAGE. A predominant fraction at $41-45,000 \mathrm{D}$ is obtained with HRF activity. Minor peaks are also seen at 7,000, 11,000-12,000, $15,000-17,000$, and $65,000-85,000$. The stacking gel corresponds to fraction 33 .

cells. In the course of such studies, it became evident that multiple molecular forms can be obtained and it is unclear whether these represent multiple molecular forms of a single gene product, or whether more than one substance possesses the same activity. Since we have demonstrated that hr-IL3 and GM-CSF possess histamine releasing activity, the likelihood that multiple cytokines will be found to possess such activity seems great. In addition, each of these may be heterogeneous as purified from stimulated cell supernatants by virtue of either aggregation, proteolysis, or carbohydrate content.

We have demonstrated that our HRF has a major form at molecular weight 30,000 (minor components may be seen at 15,000 and 50,000) when assessed by gel filtration HPLC while two species are obtained upon reverse-phase HPLC, and three forms upon Accell-QMA anion exchange-HPLC. It is of interest that Lett-Brown et al. (16) estimated that HRF has a molecular weight of 10,000-30,000 and also observed two major peaks of HRF activity upon reverse-phase HPLC, however the recovery was only $5-40 \%$. Our earlier study indicated two major forms upon QAE Sephanex-A50, however, none of these procedures proved suitable for preparative isolation and of these only the Accell-QMA anion exchange-HPLC step gives a high yield (Table I). Critical for further purification and characterization of this material was the observation that it could be recovered from SDS gels in high yield so that functional activity is retained. This is an extremely powerful method for separation from other contaminating proteins and when coupled with gel filtration HPLC and Accell-QMA anion exchange HPLC yields material that appears purified. A variety of components can be recovered for further characterization. It is important to note that material between 12,000 and 17,000 D is particularly prominent upon SDS gel electrophoresis even though the major peak upon gel filtration is 30,000 . This suggests that HRF can exist as a dimer (or higher oligomers) which are separated in the presence of SDS. It is of interest that other workers have characterized HRF from lung macrophages $(8,9)$ and nasal washings $(10)$ and reported a molecular weight of 18,000 and 15,000 . These sources of HRF have been shown to require cell bound IgE in order to cause 


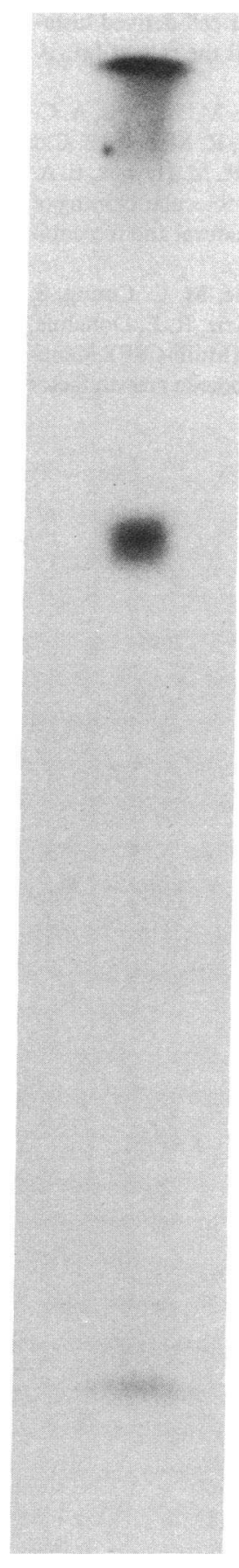

Figure 8. Autoradiogram of major HRF peak obtained upon electroelution of the Accell QMA peak III from SDS-PAGE. A single band is obtained at 41,000 . Material at the top of the gel is likely an aggregate.

histamine release $(9,10,18)$ and responsiveness to such HRF has been found limited to atopic individuals $(19,20)$ and to define a type of IgE heterogeneity. The HRF derived from mononuclear cells differs since it is active upon either nonatopic or atopic basophils (21) as is true of either IL3 or GMCSF (11). Thus far we have been unable to identify MNCHRF as being identical to any previously described cytokine. A firmer distinction from IL3 or GM-CSF will require use of antisera to these cytokines as well as antisera to HRF. Preliminary data suggest that HRF is blocked and lacks a free N-terminal, while IL3 and GM-CSF have N-terminal alanine (22,
23). However, this requires confirmation and isolation of one or more cyanogen bromide cleavage fragments of HRF in order to sequence an internal segment. Such studies are in progress.

\section{Acknowledgments}

Supported by Allergic Diseases Center grant 2 P50AI-1633710.

\section{References}

1. Ishizaka, T. 1981. Analysis of triggering events in mast cells for immunoglobulin E-mediated histamine release. J. Allergy Clin. Immunol. 67:90-96.

2. Grant, J. A., E. Dupree, A. S. Goldman, D. R. Schultz, and A. L. Jackson. 1975. Complement-mediated release of histamine from human leukocytes. J. Immunol. 114:1101-1106.

3. Kaplan, A. P., M. Haak-Frendscho, A. Fauci, C. Dinarello, and E. Halbert. 1985. A histamine-releasing factor from activated human mononuclear cells. J. Immunol. 135:2027-2032.

4. Sedgwick, J. D., L. P. G. Holt, and K. J. Turner. 1981. Production of a histamine releasing lymphokine by antigen or mitogen-stimulated human peripheral T cells. Clin. Exp. Immunol. 45:409-418.

5. Goetzl, E. J., D. W. Foster, and D. G. Payan. 1984. A basophilactivating factor from human T lymphocytes. Immunology. 53:227234.

6. Orchard, M. A., A. Kagey-Sobotka, D. Proud, and L. M. Lichtenstein. 1986. Basophil histamine release induced by a substance from stimulated platelets. J. Immunol. 136:2240-2244.

7. White, M. V., and M. A. Kaliner. 1987. Neutrophils and mast cells. I. Human neutrophil derived histamine releasing activity. J. Immunol. 139:1624-1630.

8. Schulman, E. S., M. C. Liu, D. Proud, D. W. MacGlashan, Jr., L. M. Lichtenstein, and M. Plaut. 1985. Human lung macrophages induce histamine release from basophils and mast cells. Hum. Rev. Respir. Dis. 131:230-235.

9. Liu, M. C., D. Proud, L. M. Lichtenstein, D. W. MacGlashan, Jr., R. P. Schleimer, N. F. Adkinson, Jr., A. Kagey-Sobotka, E. S. Schulman, and M. Plaut. 1986. Human lung macrophages-derived histamine-releasing activity is due to IgE-dependent factors. $\mathrm{J} . \mathrm{Im}$ munol. 136:2588-2595.

10. MacDonald, S. M., L. M. Lichtenstein, D. Proud, M. Plaut, R. M. Naclerio, D. W. MacGlashan, and A. Kagey-Sobotka. 1987. Studies of IgE-dependent histamine releasing factors: Heterogeneity of IgE. J. Immunol. 139:506-512.

11. Haak-Frendscho, M., N. Arai, K. I. Arai, M. L. Baeza, A. Finn, and A. P. Kaplan. 1988. Human recombinant granulocyte-macrophage colony-stimulating factor and interleukin 3 cause basophil histamine release. J. Clin. Invest. 82:17-20.

12. Beaven, M. A., S. Jacobsen, and Z. Horakova. 1972. Modification of the enzymatic isotopic assay of histamine and its application of measurement of histamine in tissues, serum and urine. Clin. Chem. Acta. 37:91-103.

13. Markwell, M. A. K. 1982. A new solid phase reagent to iodinate proteins. Anal. Biochem. 125:427-432.

14. Bradford, M. M. 1976. A rapid and sensitive method for the quantification of microgram quantities of protein utilizing the principle of protein-dye binding. Anal. Biochem. 72:248-254.

15. Maniatis, T., E. T. Fristsch, J. A. Sambrook. 1982. Molecular cloning. A laboratory manual. Appendix A: Biochemical Techniques. Cold Spring Harbor Laboratory, Cold Spring Harbor, NY. 466-467.

16. Laemmli, U. K. 1970. Cleavage of structural proteins during the assembly of the head of bacteriophage T4. Nature (Lond.). 227:680-685.

17. Lett-Brown, M. A., D. O. Thueson, D. E. Plank, L. Duffy, and 
J. A. Grant. 1984. Histamine-releasing activity. V. Cell Immunol. 87:445-451.

18. White, M. V., A. P. Kaplan, M. Haak-Frendscho, and M. Kaliner. 1988. Neutrophils and mast cells. Comparison of neutrophilderived histamine-releasing activity (HRA-N) with other histamine releasing factors. J. Immunol. 141:3575-3583.

19. MacDonald, S. M., A. Kagey-Sobotka, D. Proud, R. M. Naclerio, and L. M. Lichtenstein. 1987. Histamine releasing factor: release mechanism and responding population. J. Allergy Clin. Immunol. 79:248. (Abstr.)

20. Fịsher, R. H., A. Kagey-Sobotka, D. Proud, M. A. Archard, and L. M. Lichtenstein. 1987. Platelet-basophil interactions: clinical covalates. J. Allergy Clin. Immunol. 79:196. (Abstr.)

21. Baeza, M. L., M. Haak-Frendscho, S. Satnick, and A. P. Kap- lan. 1988. Responsiveness to human mononuclear cell-derived histamine releasing factor: studies of allergic status and the role of IgE. $J$. Immunol. 141:2688-2692.

22. Wong, G. G., J. S. Witek, P. A. Temple, K. M. Wilkens, A. C. Leary, D. P. Luxemberg, S. S. Jones, E. L. Brown, R. M. Kay, E. C.d Orr, C. Shoemaker, D. W. Golde, R. J. Kaufman, R. M. Hewick, E. A. Wang, and S. C. Clark. 1985. Human GM-CSF: molecular cloning of the complementary DNA and purification of the natural and recombinant proteins. Science (Wash. DC). 228:810-815.

23. Yang, Y-C., A. B. Ciarletta, P. A. Temple, M. C. Chung, S. Kovacie, J. S. Witek-Giannotti, A. C. Leary, R. Kriz, R. E. Donahue, G. G. Wong, and S. C. Clark. 1986. Human IL-3 (Multi-CSF): identification by expression cloning of a novel hematopoietic growth factor related to murine IL-3. Cell. 47:3-10. 